\title{
红壤侵蚀区芒萁对土壤可溶性有机质光谱特征的 影响
}

\section{张浩吕茂奎 谢锦升}

湿润亚热带山地生态国家重点实验室培育基地, 福州 350007; 福建师范大学地理科学学院, 福州 350007

摘 要 可溶性有机质(DOM) 是森林生态系统能量循环的主要载体, 在碳循环过程中发挥着重要的作用。为了深入了解植被 恢复后土壤DOM的变化和结构特征, 在典型红壤侵蚀区福建省龙岩市长汀县河田镇选取不同恢复年限的马尾松(Pinus massoniana) 林为研究对象, 利用光学技术对比分析了不同恢复年限(0年, 13年, 31 年)马尾松林保留芒萁(Dicranopteris dichotoma $)$ 覆盖地、去除芒萁覆盖地和林下裸地土壤DOM光谱特征。结果表明: 未治理地(Y0)、恢复13年(Y13)和恢复31年 (Y31)马尾松林芒萁覆盖地土壤可溶性有机碳(DOC)含量分别是林下裸地的7.61倍、4.83倍和5.47倍, 去除芒其一年后, 土壤 $\mathrm{DOC}$ 的含量显著下降, 但仍分别是林下裸地的 1.84 倍、4.12倍和 4.73 倍; 芒萁覆盖地土壤DOM的芳香化指数 $(A I)$ 和腐殖化指数 $(H I X)$ 均显著高于林下裸地, 而波长在 $250 \mathrm{~nm}$ 和 $365 \mathrm{~nm}$ 处的紫外可见光光度值之比 $\left(\mathrm{E}_{2}: \mathrm{E}_{3}\right)$ 的趋势与之相反, 去除芒萁一年后, $A I$ 和 $H I X$ 降低显著, 表明芒萁覆盖地土壤DOM腐殖化和芳香化程度更高, 分子量更大; 林下裸地DOM红外光谱中特征峰明 显不如林下芒萁覆盖地丰富, 其含有更多的羟基、羧酸类, 以及碳水化合物中的烷氧基等结构简单、易迁移的物质, 去除芒 其一年后, DOM红外光谱特征峰无明显变化, 表明芒萁是土壤DOM数量和结构的主要影响因素, 而这种影响是一个长期缓 慢的过程。从DOM光谱分析结果可知, 芒萁覆盖下土壤DOM的分子量更大, 结构更复杂, 易于被土壤胶粒吸附, 维持其化学 稳定, 利于土壤有机碳积累。由此可见, 芒萁在土壤有机碳积累过程中具有积极的作用。

关键词 植被恢复; 土壤可溶性有机质; 光谱学特征

引用格式: 张浩, 吕茂奎, 谢锦升 (2017). 红壤侵蚀区芒萁对土壤可溶性有机质光谱特征的影响. 植物生态学报, 41, 862-871. doi: 10.17521/cjpe.2016.0363

\section{Effect of Dicranopteris dichotoma on spectroscopic characteristic of dissolved organic matter in red soil erosion area}

ZHANG Hao, Lyu Mao-Kui, and XIE Jin-Sheng*

Cultivation Base of State Key Laboratory of Humid Subtropical Mountain Ecology, Fuzhou 350007, China; and School of Geographical Science, Fujian Normal University, Fuzhou 350007, China

\section{Abstract}

Aims Dissolved organic matter (DOM) is the most active component of organic matters in soils, and plays an important role in carbon cycles. It is a mixed organic compound with varying molecular sizes and weights. We aimed to explore the impacts of Dicranopteris dichotoma coverage on quantity and structure of DOM after vegetation restoration in severely eroded red soil region.

Methods A typical sequence of vegetation restoration (Y0, without ecological restoration; Y13, ecological restoration for 13 years; Y31, ecological restoration for 31 years) was selected as the research object in Hetian Town, Changting County, Fujian Province, China. At each experimental site, soils were subject to three treatments-NRd, not removed D. dichotoma; Rd, removed D. dichotoma; and CK, control, and the effects of $D$. dichotoma on the spectral characteristics of DOM were evaluated.

Important findings The results indicated that the quantity of soil DOC under NRd treatment of the Y0, Y13 and Y31 was 7.61, 4.83, and 5.47 times higher than their CK treatment, respectively. The Rd treatment had significantly lower DOC than that under NRd treatments, and it was $1.84,4.12$, and 4.73 times higher than their CK treatments, respectively. Thus the $D$. dichotoma had exerted significant effects on the quantity of soil DOM. The Aromaticity index $(A I)$, emission fluorescence spectrum humification index $\left(H I X_{\mathrm{em}}\right)$ and synchronous fluorescence spectrum humification index $\left(H I X_{\text {syn }}\right)$ of DOM under the NRd treatment were significantly higher than those of the CK treatments in Y13 and Y31, respectively. However, the ratio of ultraviolet-visible light absorption

收稿日期Received: 2016-11-28 接受日期Accepted: 2017-05-31

* 通信作者Author for correspondence (E-mail: jshxie@163.com) 
photometric quantity at $250 \mathrm{~nm}$ wavelength to ultraviolet-visible light absorption photometric quantity at $365 \mathrm{~nm}$ wavelength $\left(\mathrm{E}_{2}: \mathrm{E}_{3}\right)$ had an opposite trend. It showed that the DOM structure in soils covered by D. dichotoma contained more aromatic nucleus and had higher aromaticity and humification, and DOM molecular was larger. In addition, the $A I$ and humification index $(H I X)$ of DOM under the Rd treatment was significantly decreased compared with the NRd treatment. Similar results were observed by analysis of emission and synchronous fluorescence spectrum, and by the Fourier infrared transmission spectrum analysis. These results suggest that $D$. dichotoma had positive impacts on the complexity of DOM structure, but it was a long and slow process. The DOM spectral analysis showed that the soil DOM covered by D. dichotoma had a stable and complex structure and was easily adsorbed by soil colloid. As a result, Dicranopteris dichotoma had a positive effect on the accumulation of soil organic carbon.

Key words vegetation restoration; dissolved organic matter; spectroscopic characteristic

Citation: Zhang H, Lyu MK, Xie JS (2017). Effect of Dicranopteris dichotoma on spectroscopic characteristic of dissolved organic matter in red soil erosion area. Chinese Journal of Plant Ecology, 41, 862-871. doi: 10.17521/cjpe.2016.0363

可溶性有机质(DOM) 是指能够通过 $0.45 \mu \mathrm{m}$ 篮 孔且溶于水、酸或碱溶液的不同分子大小和有机结 构的混合体(Michalzik \& Matzner, 1999; 杨玉盛等, 2003)。它不仅影响着森林生态系统的能量交换, 而 且直接参与生物地球化学循环, 尤其在全球碳循环 过程中担当着重要角色(Kalbitz et al., 2007)。由于 DOM在土壤有机质中的比例很小 $(<2 \%)$ (Andreasson et al., 2009), 在过去的碳循环研究中常被忽 略, 但因其动态变化可以灵敏地反映土壤有机碳循 环与平衡趋势(焦坤和李忠佩, 2005), 故而在森林生 态系统中, DOM的来源、通量和迁移特征及其对土 壤有机质的贡献受到重点关注(Schwendenmann \& Veldkamp, 2005; Chang et al., 2007; Fujii et al., 2009; Carter et al., 2012), 如一些研究表明DOM对陆地生 态系统的净生产力贡献高达 11\%-17\% (Gielen et al., $2011), 20 \%-50 \%$ 的森林土壤有机质可能来自 DOM (Kalbitz \& Kaiser, 2008), 在湿润的气候条件下, DOM对深层土壤有机质的贡献可能更大(Mccarthy, 2005), 但是林下植被对土壤DOM结构特征的影响 仍不清楚。南方红壤侵蚀区植被恢复后, 林下分布 着大片芒萁(Dicranopteris dichotoma)群落, 那么芒 其对土壤DOM结构特征影响如何? 随着植被恢复, 在土壤有机碳积累过程中芒萁担任何种角色? 目前 对之尚不清楚。

目前关于DOM结构特征的研究采用的手段主 要是光学技术, 其简单易操作, 不会对样品产生破 坏, 而且分析高效, 分析结果所含信息丰富, 因此 在DOM微观结构的研究中得到了广泛的应用。最为 常用的就是紫外可见分光光谱和苂光光谱分析技术, 另外, 还有很多研究利用傅里叶变换红外光谱测定
DOM的特征官能团，更为详细地表征其结构特性 (周江敏等, 2004; Carter et al., 2012; 刘翥等, 2015)。

鉴于此, 本研究在典型红壤侵蚀区福建省龙岩 市长汀县河田镇选取不同恢复年限 $(0$ 年, 13 年, 31 年) 的马尾松林为研究对象, 利用光学技术, 通过对比 分析不同恢复年限马尾松林保留芒萁覆盖地、去除 芒萁覆盖地和林下裸地土壤DOM光谱特征, 旨在 分析植被恢复过程中林下植被芒萁对土壤DOM结 构特征的影响, 揭示其在土壤有机碳积累过程中担 任的角色, 为进一步理解植被恢复过程中土壤有 机碳积累的机制提供科学依据。

\section{1 材料和方法}

\section{1 研究区和试验地概况}

研究区位于福建省龙岩市长汀县河田镇 $\left(116.30^{\circ}-116.52^{\circ} \mathrm{E}, 25.55^{\circ}-25.80^{\circ} \mathrm{N}\right)$, 该区地处福建 省西南部, 属中亚热带季风气候, 年降水量 $1737 \mathrm{~mm}$, 年蒸发量 $1403 \mathrm{~mm}$, 年平均气温 $17.5-18.8{ }^{\circ} \mathrm{C}$, 平均 无霜期 260 天, 年平均日照时间为 $1924.6 \mathrm{~h}, \geqslant 10{ }^{\circ} \mathrm{C}$ 积温为4 100-4 $650{ }^{\circ} \mathrm{C}$ 。该区属于河谷盆地, 四周被 低山高丘环抱, 海拔在300-500 m之间。该区原有地 带性植被(常绿阔叶林)基本被破坏殆尽, 现有植被 主要以马尾松次生林和人工林为主, 林下植被以芒 萁为主。该区土壤为燕山运动早期形成的中粗粒花 岗岩发育的红壤, 可蚀性高, 花岗岩风化壳深厚, 结构松散, 降雨侵蚀严重, 加之历史上该区植被破 坏严重, 使河田镇成为全国水土流失极为严重的区 域, 许多地方表土层丧失殆尽, 植被恢复困难。

采用时空代换方案, 在红壤典型侵蚀区福建省 龙岩市长汀县河田镇选择土壤母岩均为花岗岩的未 
治理地、恢复13年和31年的马尾松林地(表1)组成植 被恢复年限序列。恢复13年和31年的马尾松林地治 理前均为表土层土壤流失殆尽，心土层出露，本底 条件与未治理地基本一致。

\section{2 样地调查和样品采集处理}

于2014年 8 月上旬，在每个林地设置3种处理: 保留芒萁覆盖、去除芒萁覆盖以及与之毗邻的林下 未生长芒其的裸露地。设置 3 个 $20 \mathrm{~m} \times 20 \mathrm{~m}$ 的标准 样地, 每个标准样地分成 4 个 $10 \mathrm{~m} \times 10 \mathrm{~m}$ 的亚样方, 随机选取一个亚样方作为保留芒萁覆盖处理, 1 个亚 样方作为去除芒萁覆盖处理, 与之毗邻的林下裸地 作为对照处理, 其面积和形状依每个样地实际裸露 情况确定, 面积不少于 $100 \mathrm{~m}^{2}$ 。在去除芒萁一年后, 即 2015 年 8 月中旬, 用取土钻 (内径为 $5 \mathrm{~cm}$ ) 在每个处 理样地内取 0-10 $\mathrm{cm}$ 土层的土壤, 按 $\mathrm{S}$ 型随机多点 (15个点)取样并混合。取回的部分新鲜土样在实验 室拣去石砾、植物根系和大于 $2 \mathrm{~mm}$ 的碎屑, 风干后 过 $0.149 \mathrm{~mm}$ 土壤篎, 存于密闭自封袋中, 用于土壤 理化性质和土壤有机碳的测定。剩余的新鲜土样过 $2 \mathrm{~mm}$ 土壤篮, 冷冻于 $-4{ }^{\circ} \mathrm{C}$ 冰箱中, 用于土壤DOM 的浸提。

\section{3 实验方法}

土壤理化性质测定：土壤有机碳(SOC)和土壤
全氮(TN)采用碳氮元素分析仪(Vario EL Max CN, Elementar Analysensystem GmbH, Langenselbold, Germany)测定，pH值使用CHN868型 $\mathrm{pH}$ 计(CHN868， Thermom, Orion, USA)测定。

土壤DOM浸提：样品DOM采用水浸提法 $(\mathrm{Wu}$ et al., 2010), 取 $10 \mathrm{~g}$ 鲜土于 $50 \mathrm{~mL}$ 离心管中, 加入 去离子水 $40 \mathrm{~mL}$ (水土比 $4: 1, \mathrm{~V}: \mathrm{W}$ ), 振荡 $30 \mathrm{~min}$ $\left(260 \mathrm{r} \cdot \mathrm{min}^{-1}\right)$, 离心 $10 \mathrm{~min}\left(4000 \mathrm{r} \cdot \mathrm{min}^{-1}\right)$ 后, 用 $0.45 \mu \mathrm{m}$ 滤膜过滤，滤液用于DOC测定。

DOC浓度测定：采用岛津 TOC-V CPH有机碳 分析仪(TOC-V CPH, Shimadzu, Japan)测定样品DOC 浓度。

土壤DOM紫外光谱测定：用紫外可见分光光 度计(UV-2450, Shimadzu, Kyoto, Japan)测定波长为 $250 、 254$ 和 $365 \mathrm{~nm}$ 处的吸光值。

土壤 $\mathrm{DOM}$ 荧光光谱测定: 荧光光谱使用日立 F7000仪器(F7000, Hitachi, Tokyo, Japan)进行测定, 激发和发射光柱狭缝宽度都为 $10 \mathrm{~nm}$, 扫描速度 $1200 \mathrm{~nm} \cdot \mathrm{min}^{-1}$, 激发波长 $254 \mathrm{~nm}$, 苂光发射光谱波长 范围300-480 nm, 苂光同步光谱波长范围250-500 nm。

为了提高灵敏度, 紫外可见分光光度计吸收值 测定和荧光光谱扫描前, 待测液均用 $2 \mathrm{~mol} \cdot \mathrm{L}^{-1} \mathrm{HCl}$ 将其 $\mathrm{pH}$ 值调至2。

表1 样地基本概况(平均值土标准偏差)

Table 1 The basic background characteristics of sample plot (mean $\pm S D$ )

\begin{tabular}{|c|c|c|c|c|c|c|c|c|c|}
\hline \multirow{3}{*}{ 因子 Factor } & \multicolumn{9}{|c|}{ 林分类型 Stand type } \\
\hline & \multicolumn{3}{|c|}{ Y0 } & \multicolumn{3}{|c|}{ Y13 } & \multicolumn{3}{|c|}{ Y31 } \\
\hline & NRd & $\mathrm{Rd}$ & $\mathrm{CK}$ & NRd & $\mathrm{Rd}$ & $\mathrm{CK}$ & NRd & $\mathrm{Rd}$ & CK \\
\hline 治理历史 & \multicolumn{3}{|c|}{ 土壤侵蚀严重 } & \multicolumn{3}{|c|}{ 从2002年开始治理 } & \multicolumn{3}{|c|}{ 从1984年开始治理 } \\
\hline Management process & \multicolumn{3}{|c|}{ Suffer from serious soil erosion } & \multicolumn{3}{|c|}{ Ecological restoration since from 2002} & \multicolumn{3}{|c|}{ Ecological restoration since from 1984} \\
\hline 优势种 & \multirow{2}{*}{\multicolumn{3}{|c|}{$\begin{array}{l}\text { 马尾松, 芒萁 } \\
\text { Pinus massoniana, } \\
\text { Dicranopteris dichotoma }\end{array}$}} & \multirow{2}{*}{\multicolumn{3}{|c|}{$\begin{array}{l}\text { 马尾松, 芒萁 } \\
\text { Pinus massoniana, } \\
\text { Dicranopteris dichotoma }\end{array}$}} & \multirow{2}{*}{\multicolumn{3}{|c|}{$\begin{array}{l}\text { 马尾松, 芒萁 } \\
\text { Pinus massoniana, } \\
\text { Dicranopteris dichotoma }\end{array}$}} \\
\hline Dominant species & & & & & & & & & \\
\hline $\begin{array}{l}\text { 平均树高 } \\
\text { Average height of trees (m) }\end{array}$ & \multicolumn{3}{|l|}{2} & \multicolumn{3}{|l|}{7} & \multicolumn{3}{|l|}{13.7} \\
\hline $\begin{array}{l}\text { 平均胸径 Average } \\
\text { diameter at breast height }(\mathrm{cm})\end{array}$ & \multicolumn{3}{|l|}{3.1} & \multicolumn{3}{|l|}{7.4} & \multicolumn{3}{|l|}{14} \\
\hline $\begin{array}{l}\text { 植株密度 } \\
\text { Plant density }\left(\text { stems } \cdot \mathrm{hm}^{-2} \text { ) }\right.\end{array}$ & \multicolumn{3}{|l|}{1741} & \multicolumn{3}{|l|}{3341} & \multicolumn{3}{|l|}{1433} \\
\hline $\begin{array}{l}\text { 芒萁覆盖度 Coverage of } \\
\text { Dicranopteris dichotoma }(\%)\end{array}$ & \multicolumn{3}{|l|}{15} & \multicolumn{3}{|l|}{90} & \multicolumn{3}{|l|}{85} \\
\hline 坡度 Slope $\left({ }^{\circ}\right)$ & \multicolumn{3}{|l|}{19} & \multicolumn{3}{|l|}{8} & \multicolumn{3}{|l|}{11} \\
\hline $\begin{array}{l}\text { 土壤有机碳 } \\
\text { Soil organic carbon }\left(\mathrm{g} \cdot \mathrm{kg}^{-1}\right)\end{array}$ & $6.37 \pm 0.12$ & $3.42 \pm 0.01$ & $1.60 \pm 0.14$ & $10.16 \pm 1.47$ & $11.30 \pm 1.28$ & $8.09 \pm 0.52$ & $14.33 \pm 4.78$ & $14.23 \pm 1.94$ & $5.32 \pm 0.54$ \\
\hline 总氮Total nitrogen $\left(\mathrm{g} \cdot \mathrm{kg}^{-1}\right)$ & $0.61 \pm 0.02$ & $0.52 \pm 0.19$ & $0.26 \pm 0.07$ & $0.74 \pm 0.04$ & $0.66 \pm 0.01$ & $0.48 \pm 0.09$ & $0.91 \pm 0.25$ & $0.81 \pm 0.02$ & $0.36 \pm 0.16$ \\
\hline $\mathrm{pH}$ & $4.37 \pm 0.07$ & $4.58 \pm 4.58$ & $4.67 \pm 0.07$ & $4.19 \pm 0.07$ & $4.39 \pm 0.07$ & $4.50 \pm 0.04$ & $4.24 \pm 0.06$ & $4.44 \pm 0.03$ & $4.69 \pm 0.14$ \\
\hline
\end{tabular}

CK, 林下裸地; NRd, 芒萁覆盖地; Rd, 去除芒萁覆盖地。Y 0 , 未治理地; Y13, 恢复 13 年马尾松林地; Y31, 恢复31年马尾松林地。

CK, control; NRd, not removed Dicranopteris dichotoma; Rd, removed D. dichotoma. Y0, without ecological restoration; Y13, ecological restoration for 13 years; Y31, ecological restoration for 31 years.

www.plant-ecology.com 
土壤 DOM 红外光谱测定: 用FTIR 光谱仪 (Nicolet Magna FTIR 550, Thermo, Waltham, USA) 测定红外光谱, 将 $1 \mathrm{mg}$ 冷冻干燥的土壤DOM样品 与 $400 \mathrm{mg}$ 干燥的 $\mathrm{KBr}$ (光谱纯)磨细混匀, 在 $10 \mathrm{t} \cdot \mathrm{cm}^{-2}$ 压强下压成薄片并维持 $1 \mathrm{~min}$, 测定并记录其光谱, 波谱扫描范围为4 000-400 $\mathrm{cm}^{-1}$ 。

\section{4 计算方法和数据处理}

使用UV-2450 (岛津)紫外可见分光光度计检测 待测液在波长 $254 \mathrm{~nm}$ 处的吸收值来表征其芳香化 程度(Akagi et al., 2007; Wu et al., 2010), 即芳香化 指数 $(A I)$ 。

$\mathrm{E}_{2}: \mathrm{E}_{3}$ 值表征待测样中DOM平均分子量的大小, 该值越大表明平均分子量越小, 计算公式为 $\mathrm{E}_{2}: \mathrm{E}_{3}=$ $\mathrm{UV}_{250}: \mathrm{UV}_{365}$ (Peuravuori \& Pihlaja, 1997)。

苂光发射光谱中 $\Sigma 435-480 \mathrm{~nm}$ 区域与 $\Sigma 300$ $345 \mathrm{~nm}$ 区域的峰面积比值被称为荧光发射光谱腐 殖化指数 $\left(H I X_{\mathrm{em}}\right)$, 苂光同步光谱波长 460 与 $345 \mathrm{~nm}$ 处苂光强度的比值被称为菼光同步光谱腐殖化指数 $\left(H I X_{\text {syn }}\right)($ Kalbitz et al., 1999)。

所有数据通过软件Excel 2013进行统计处理, 利用软件SPSS 19.0进行方差分析和皮尔逊相关分 析，相关图表全部用Origin 9.0软件完成。

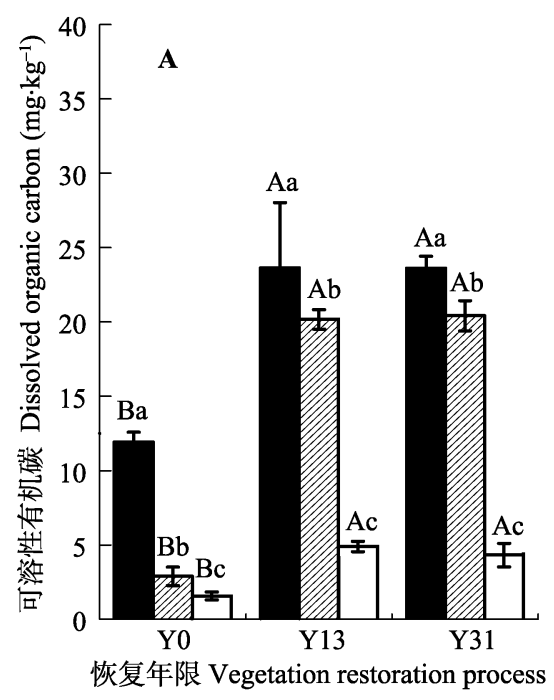

NRd

\section{2 结果和分析}

\section{1 马尾松林土壤DOM的数量特征}

本研究采用土壤可溶性有机碳(DOC)的含量来 表征土壤中DOM的数量。Y0、Y13、Y31马尾松林 芒萁覆盖地土壤DOC含量分别是林下裸地的 7.61 倍、4.83倍、5.47倍(图1A)，去除芒萁一年后，土壤 DOC的含量显著下降, 分别是林下裸地的 1.84 倍、

4.12倍、4.73倍 $(p<0.05)$, 表明芒萁对土壤DOM数 量有显著影响。

$\mathrm{DOC}$ 在土壤总有机碳(SOC)中分配比例的变化 趋势同土壤DOC含量的变化基本相同(图1B), Y0马 尾松林林下裸地显著低于芒萁覆盖地 $(p<0.05)$, 而 去除芒萁处理一年后其比例已经和林下裸地没有明 显差异, 同样, Y13和Y31保留芒萁处理的DOC占 $\mathrm{SOC}$ 的比例均显著高于林下裸地，且去除芒萁处理 一年后其比例已经显著降低，但仍显著高于林下裸 地 $(p<0.05)$, 显然, 芒萁覆盖增加了DOC在 SOC中 的分配比例。

\section{2 马尾松林土壤DOM的紫外光谱特征}

$\mathrm{Y} 0$ 马尾松林林下裸地土壤DOM的 $A I$ 显著小于 芒萁覆盖地 $(p<0.05)$, 去除芒萁一年后，已和林下 裸地无差异(图2A); 同样，Y13和Y31马尾松林林下

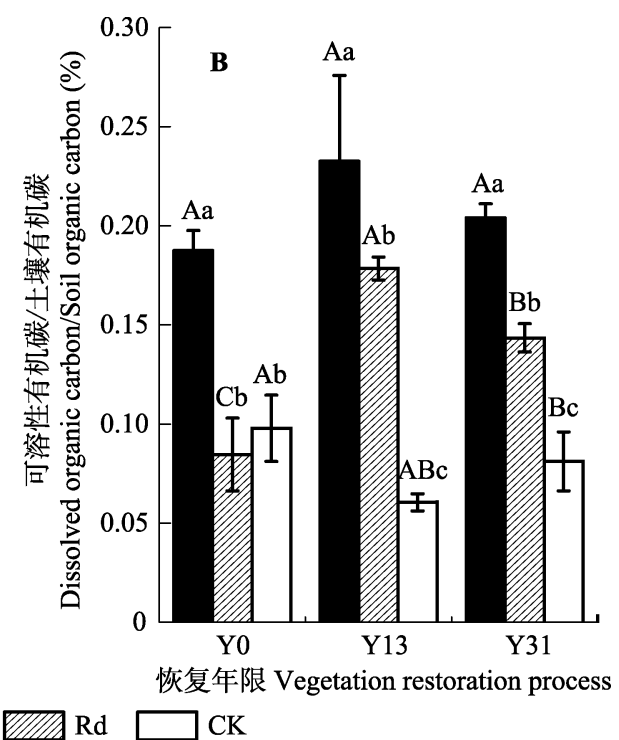

图1 土壤可溶性有机质(DOM)数量变化(平均值标准偏差)。不同小写字母表示同一恢复年限不同处理间各指标间差异显著 $(p<0.05)$; 不同大写字母表示同一处理不同恢复年限间各指标间差异显著 $(p<0.05)$ 。CK，林下裸地; NRd，芒其覆盖地; Rd， 去除芒其覆盖地。Y0，未治理地；Y13，恢复13年马尾松林地；Y31，恢复31年马尾松林地。

Fig. 1 Variation of the quantity of soil dissolved organic matter (DOM) (mean $\pm S D$ ). Different lowercase letters stand for the significant difference between different experimental treatments in the same vegetation restoration years $(p<0.05)$. Different capitals stand for the significant difference between the different vegetation restoration years in the same experimental treatment $(p<0.05)$. CK, control; NRd, not removed Dicranopteris dichotoma; Rd, removed D. dichotoma. Y0, without ecological restoration; Y13, ecological restoration for 13 years; Y31, ecological restoration for 31 years. 
裸地土壤 $\mathrm{DOM}$ 的 $A I$ 值均显著低于芒萁覆盖地, 且 去除芒其一年后, 其 $A I$ 值显著降低, 但仍显著高于 林下裸地 $(p<0.05)$, 这些结果表明, 去除芒其后土 壤DOM的腐殖化程度逐渐接近林下裸地, 证明芒 其对维持土壤 DOM的芳香化程度具有明显作用, 而且随着马尾松林恢复年限增加, 芒萁覆盖地土壤 $\mathrm{DOM}$ 的芳香化程度显著增加，而林下裸地没有明 显差异, 表明芒其是控制土壤DOM腐殖化程度的 主要因素。

$\mathrm{Y} 0$ 马尾松林林下裸地 $\mathrm{E}_{2}: \mathrm{E}_{3}$ 明显高于芒萁覆盖 地, 且去除芒萁一年后 $\mathrm{E}_{2}: \mathrm{E}_{3}$ 也显著升高, 有接近林 下裸地的趋势, 但仍明显低于林下裸地 $(p<0.05)$ (图2B)。同样, $\mathrm{Y} 13$ 和 $\mathrm{Y} 31$ 马尾松林林下裸地 $\mathrm{E}_{2}$ : $\mathrm{E}_{3}$ 明 显高于芒其覆盖地, 但去除芒萁一年后 $\mathrm{E}_{2}: \mathrm{E}_{3}$ 与芒萁 覆盖地无差异, 以上结果表明芒萁覆盖地土壤 $\mathrm{DOM}$ 平均分子量明显大于林下裸地。另外, Y13和 $\mathrm{Y} 31$ 马尾松林芒其覆盖地土壤 $\mathrm{E}_{2}: \mathrm{E}_{3}$ 显著低于 $\mathrm{Y} 0$ $(p<0.05)$, 而这种差异在去除芒萁和林下裸地处理 上表现尤为显著, 这意味着芒萁是影响土壤 $\mathrm{DOM}$ 分子量大小的主要因素。

\section{3 马尾松林土壤DOM的荧光光谱特征}

不同恢复年限马尾松林不同处理下的土壤DOM

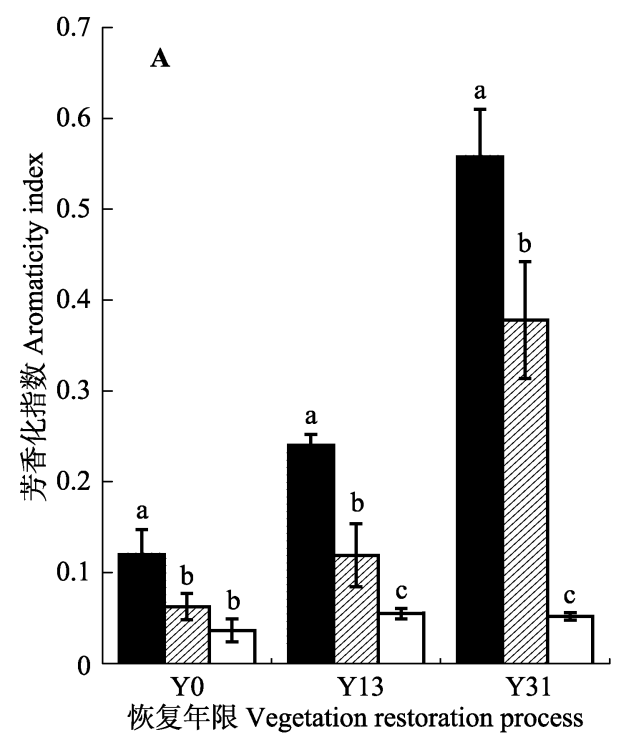

NRd
苂光发射光谱峰型图相似(图3), 但特征峰苂光强度 差异明显, 特别是不同恢复年限的马尾松林林下植 被芒萁被去除一年后，土壤DOM各基团在对应波 长上荧光强度明显降低, 意味着去除芒其后土壤 DOM结构中苂光基团在减少。Y0和Y13马尾松林芒 其覆盖地、去除芒萁地、林下裸地的土壤DOM的波 峰对应的波长主要集中在340-355 nm之间, 未见明 显的波峰位移显现, 而Y 31 马尾松林林下植被的不 同处理引起了土壤DOM波峰的明显位移, 芒萁覆 盖地DOM的波峰位于发射苂光光谱波长 $\left(\lambda_{\mathrm{em}}\right)=408$ $\mathrm{nm}$ 处, 而林下裸地的波峰位于 $\lambda_{\mathrm{em}}=353 \mathrm{~nm}$ 处, 去 除芒萁一年后, DOM的波峰位于 $\lambda_{\mathrm{em}}=402 \mathrm{~nm}$ 处, 这 意味着芒萁覆盖地土壤DOM分子内的共轭体系更 多。另外, Y31马尾松林土壤DOM苂光光谱波峰位 移明显, $\mathrm{Y} 0$ 和Y13马尾松林却未发现土壤DOM苂光 光谱波峰明显位移, 表明芒萁覆盖对DOM分子结 构的影响是一个长期缓慢的过程。

不同恢复年限的马尾松林在不同处理下的土 壤DOM菼光同步光谱峰型图相似(图4), 但不同恢 复年限、不同处理间特征峰出现的位置大致相同, 相对峰面积却不同。土壤DOM苂光同步光谱特征峰 中: 同步苂光光谱波长 $\left(\lambda_{\mathrm{syn}}\right)$ 在280-290 $\mathrm{nm}$ 处表

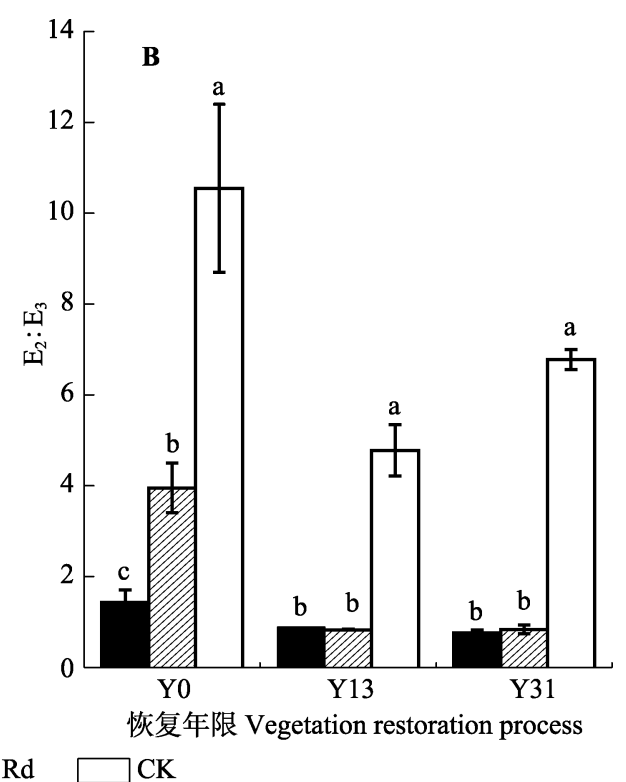

图2 土壤可溶性有机质(DOM)的紫外光谱特征(平均值土标准偏差)。不同小写字母表示同一恢复年限不同处理间各指标间差 异显著 $(p<0.05)$ 。CK, 林下裸地; NRd, 芒其覆盖地; Rd, 去除芒其覆盖地。Y 0 , 未治理地; Y13, 恢复13年马尾松林地; Y 31 , 恢复 31 年马尾松林地。 $\mathrm{E}_{2}: \mathrm{E}_{3}$, 波长在 $250 \mathrm{~nm}$ 和 $365 \mathrm{~nm}$ 处的紫外可见光光度值之比。

Fig. 2 Characteristics of soil dissolved organic matter (DOM) ultraviolet spectrum (mean $\pm S D$ ). Different lowercase letters stand for the significant difference between different experimental treatments in the same vegetation restoration years $(p<0.05)$. CK, control; NRd, not removed Dicranopteris dichotoma; Rd, removed D. dichotoma. Y0, without ecological restoration; Y13, ecological restoration for 13 years; Y31, ecological restoration for 31 years. $E_{2}: E_{3}$, the ratio of ultraviolet-visible light absorption photometric quantity at $250 \mathrm{~nm}$ wavelength to ultraviolet-visible light absorption photometric quantity at $365 \mathrm{~nm}$ wavelength.

www.plant-ecology.com 

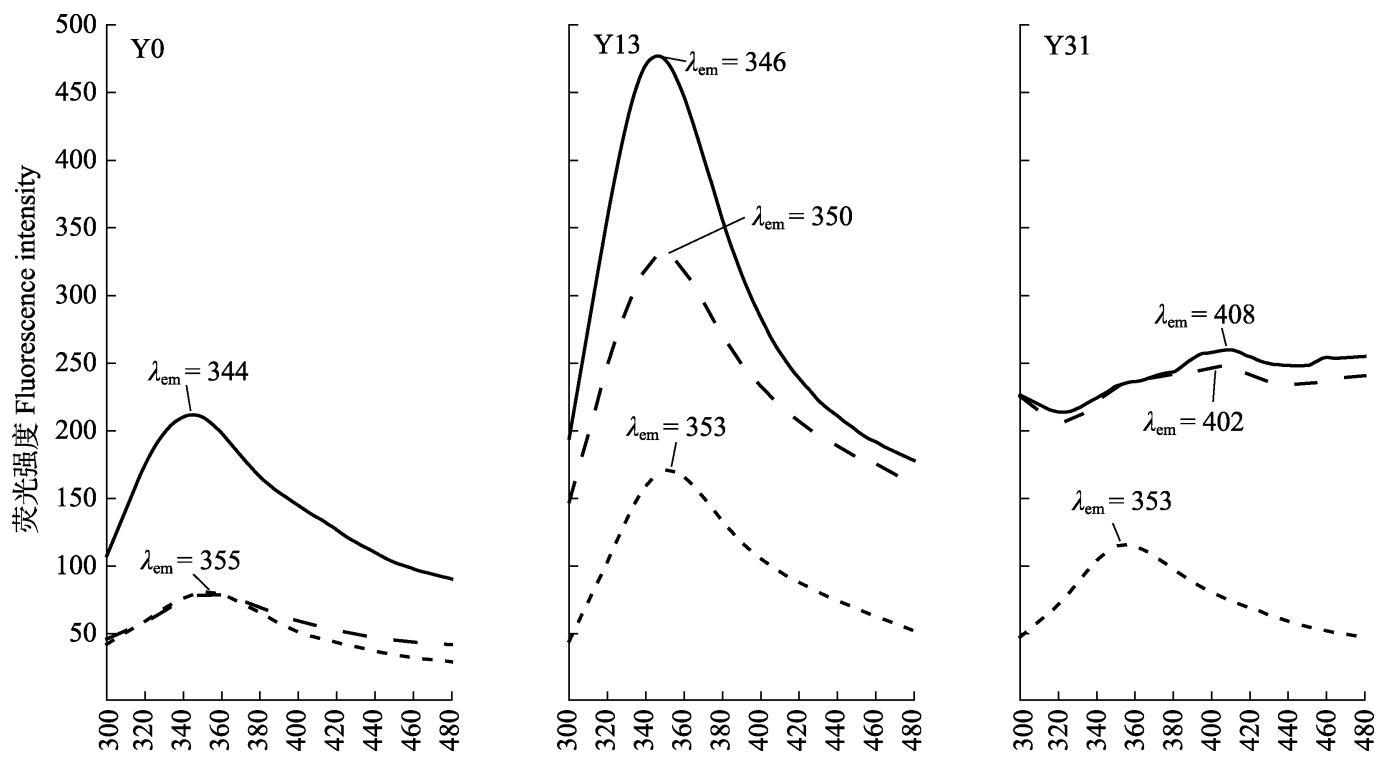

发射波长 Emission wavelength (nm)

-NRd - - Rd -- CK

图3 土壤可溶性有机质(DOM)苂光发射光谱特征。CK, 林下裸地; NRd, 芒萁覆盖地; Rd, 去除芒萁覆盖地。Y 0 , 未治理地; $\mathrm{Y} 13$, 恢复13年马尾松林地; Y31，恢复31年马尾松林地。 $\lambda_{\mathrm{em}}$, 发射荧光光谱波长。

Fig. 3 Characteristics of dissolved organic matter (DOM) emission fluorescence spectrum. CK, control; NRd, not removed Dicranopteris dichotoma; Rd, removed D. dichotoma. Y0, without ecological restoration; Y13, ecological restoration for 13 years; Y31, ecological restoration for 31 years. $\lambda_{\mathrm{em}}$, emission fluorescence spectrum wavelength.
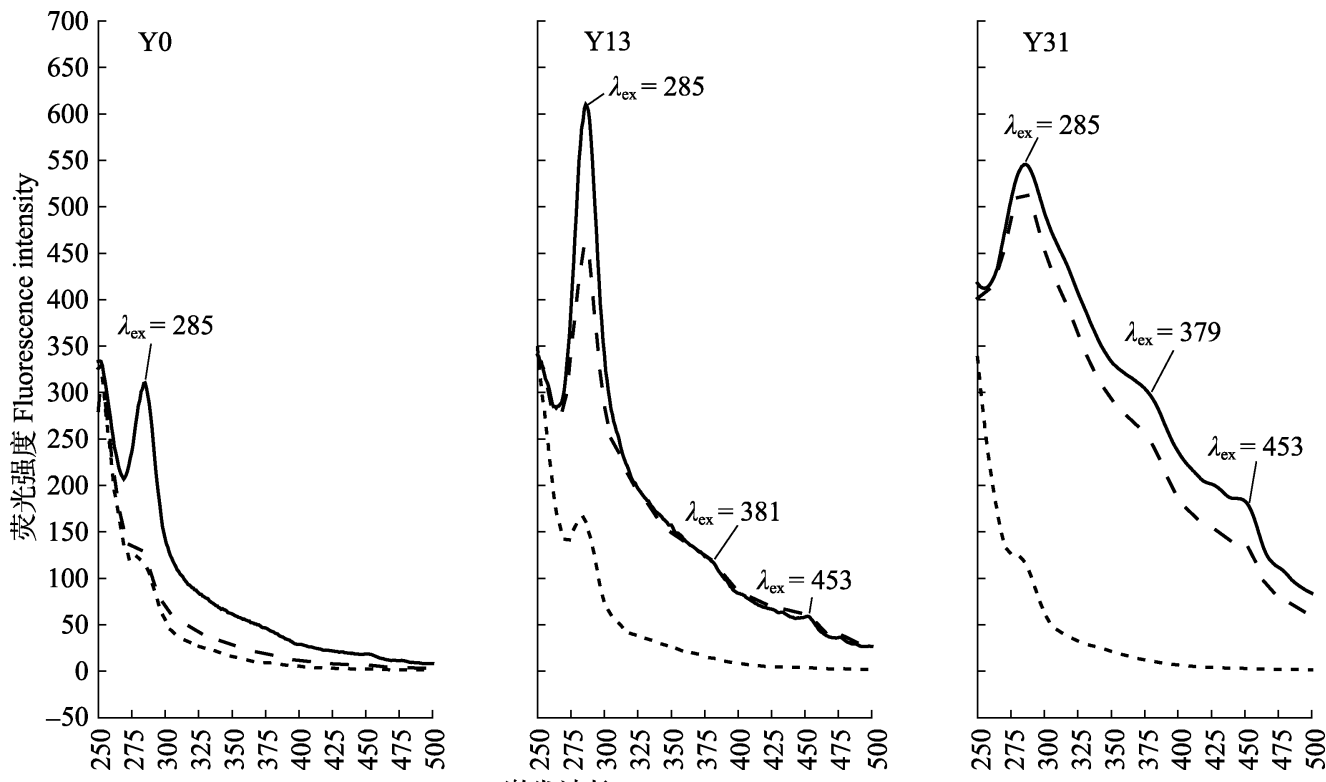

激发波长 Excitation wavelength (nm)

- NRd - - Rd - - CK

图4 土壤可溶性有机质(DOM)的苂光同步光谱特征。CK, 林下裸地; $\mathrm{NRd}$, 芒萁覆盖地; Rd, 去除芒萁覆盖地。Y0, 未治理 地; Y13，恢复13年马尾松林地; Y31，恢复31年马尾松林地。 $\lambda_{\mathrm{syn}}$, 同步荧光光谱波长。

Fig. 4 Characteristics of dissolved organic matter (DOM) synchronization fluorescence spectrum. CK, control; NRd, not removed Dicranopteris dichotoma; Rd, removed Dicranopteris dichotoma. Y0, without ecological restoration; Y13, ecological restoration for 13 years; Y31, ecological restoration for 31 years. $\lambda_{\text {syn, }}$ synchronous fluorescence spectrum wavelength.

征DOM分子中的蛋白质基团(如芳香氨基酸), 在 375-385 nm处表征DOM分子中的芳香性脂肪基团, 在450-490 nm处表征DOM分子中的木质素基团，
而各类峰的相对面积大小表征分子个基团含量的多 少(刘者等, 2015)。Y0马尾松林芒萁覆盖地土壤 $\mathrm{DOM}$ 荧光同步光谱在 $\lambda_{\mathrm{syn}}=285 \mathrm{~nm}$ 附近的相对峰面 
积明显大于林下裸地，去除芒萁一年后，该区的相 对峰面积与裸地基本相同; 同样, Y13马尾松林芒萁 覆盖地土壤DOM苂光同步光谱在 $\lambda_{\mathrm{syn}}$ 为 $285 、 381$ 和 $453 \mathrm{~nm}$ 附近的相对峰面积均大于林下裸地, 去除芒 萁一年后, 在 $\lambda_{\mathrm{syn}}=285 \mathrm{~nm}$ 附近的峰面积明显减小, 但仍大于林下裸地, 而 $\lambda_{\mathrm{syn}}$ 为 381 和 $453 \mathrm{~nm}$ 附近的相 对峰面积与林下裸地几乎无差别, 但仍大于林下 裸地; Y31马尾松林与之相似, 芒萁覆盖地土壤 $\mathrm{DOM}$ 在 $\lambda_{\mathrm{syn}}$ 为 285 、379和 $453 \mathrm{~nm}$ 附近的相对峰面积 均大于林下裸地, 去除芒其一年后, 该区对应特征 峰面积均有明显的减小趋势, 但仍大于林下裸地, 这表明芒萁对土壤中DOM分子内的蛋白质基团、芳 香性脂肪酸基团和木质素基团的维持有重要的作用, 并且在Y0马尾松林这种不稳定的生态系统中这种 作用表现得更为明显。图4还表明, 随着恢复年限的 不断增加, 土壤DOM的特征峰在不断增加, 芒萁覆 盖地表现尤为明显, 说明芒萁是影响土壤DOM分子 复杂程度的主要因素。

$\mathrm{Y} 0$ 马尾松林林下裸地土壤 $\mathrm{DOM}$ 的 $H I X_{\mathrm{em}}$ 和 $H I X_{\mathrm{syn}}$ 均显著低于芒其覆盖地 $(p<0.05)$, 去除芒萁 一年后, 土壤的 $H I X_{\mathrm{em}}$ 无明显变化, 但仍显著高于
林下裸地 $(p<0.05)$, 而 $H I X_{\mathrm{syn}}$ 显著低于芒萁覆盖地 $(p<0.05)$, 与林下裸地接近(图5); Y13马尾松林下 裸地土壤 $\mathrm{DOM}$ 的 $H I X_{\mathrm{em}}$ 和 $H I X_{\mathrm{syn}}$ 均显著低于芒萁覆 盖地 $(p<0.05)$, 去除芒其一年后, $H I X_{\mathrm{em}}$ 和 $H I X_{\mathrm{syn}}$ 变 化不明显，却仍显著高于林下裸地 $(p<0.05) ; \mathrm{Y} 31$ 马尾松林下裸地土壤 $\mathrm{DOM}$ 的 $H I X_{\mathrm{em}}$ 和 $H I X_{\mathrm{syn}}$ 显著低 于芒萁覆盖地 $(p<0.05)$, 去除芒萁一年后, 土壤 $\mathrm{DOM}$ 的 $H I X_{\mathrm{em}}$ 接近林下裸地的趋势明显, $H I X_{\mathrm{syn}}$ 显著 降低, 但仍明显高于林下裸地 $(p<0.05)$, 显然, 芒 萁覆盖地土壤DOM分子腐殖化程度更高, 分子结 构更复杂, 去除芒其一年后, 土壤DOM分子的腐殖 化程度均有不同程度的降低, 这意味着芒萁对土壤 DOM腐殖化程度的维持有深刻影响, 并且这个影 响过程是一个长期缓慢的过程。

\section{4 马尾松林土壤DOM的红外光谱特征}

土壤DOM存在 5 类特征峰: $4000-3000 \mathrm{~cm}^{-1}$ (与氢键键合的一 $\mathrm{COOH}$ 、醇、苯酚中的一 $\mathrm{OH}$ 伸缩 振动吸收峰)、1625-1690 $\mathrm{cm}^{-1}$ (苯环、烯烃类和分 子间或分子内形成氢键的羧酸中 $-\mathrm{C}=\mathrm{O}$ 的伸缩振 动峰)、1 600-1 $425 \mathrm{~cm}^{-1}$ (苯环上 $\mathrm{C}=\mathrm{C}$ 骨架振动)、 $1000-1250 \mathrm{~cm}^{-1}$ (多糖类、醇类、羧酸类及脂类

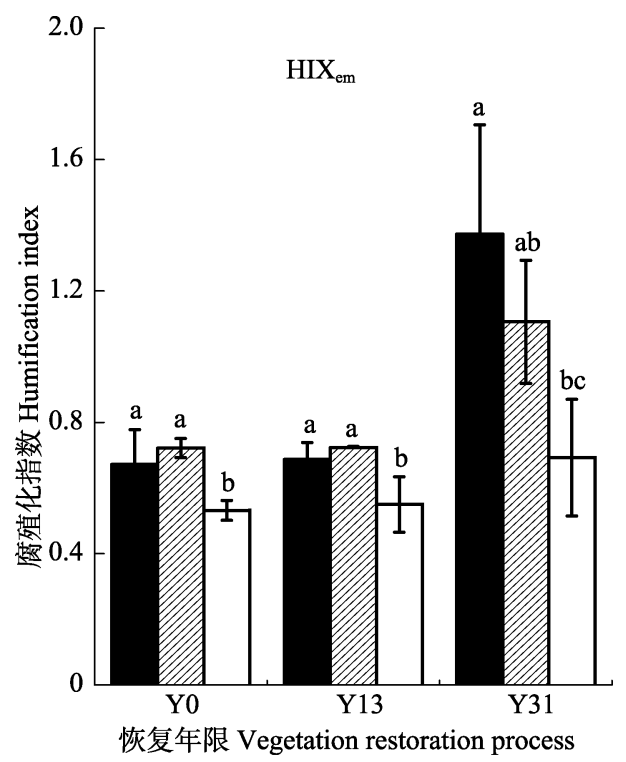

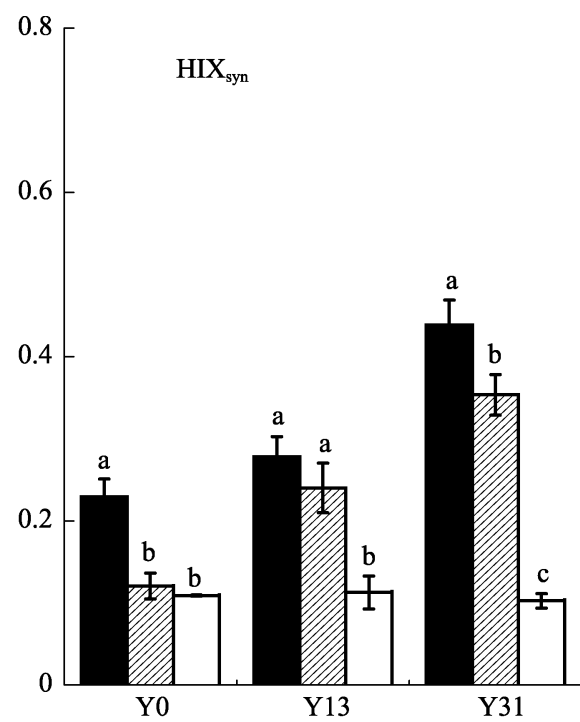

恢复年限 Vegetation restoration process

NRd

Rd

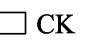

图5 土壤可溶性有机质(DOM)的苂光光谱特征(平均值土标准偏差)。不同小写字母表示同一恢复年限不同处理间各指标间差 异显著 $(p<0.05)$ 。CK, 林下裸地; NRd, 芒萁覆盖地; Rd, 去除芒萁覆盖地。Y 0 , 未治理地; Y13, 恢复13年马尾松林地; Y31, 恢复31年马尾松林地。HIX $X_{\mathrm{em}}$, 发射苂光光谱腐殖化指数; $H I X_{\mathrm{syn}}$, 同步荧光光谱腐殖化指数。

Fig. 5 Characteristics of dissolved organic matter (DOM) fluorescence spectrum (mean $\pm S D$ ). Different lowercase letters stand for the significant difference between different experimental treatments in the same vegetation restoration years $(p<0.05)$. CK, control; NRd, not removed Dicranopteris dichotoma; Rd, removed D. dichotoma. Y0, without ecological restoration; Y13, ecological restoration for 13 years; Y31, ecological restoration for 31 years. $H I X_{\text {em }}$, emission fluorescence spectrum humification index; $H I X_{\text {syn }}$, synchronous fluorescence spectrum humification index.

www.plant-ecology.com 
$-\mathrm{C}$ - $\mathrm{O}$ 一伸缩振动吸收峰)、 $460-680 \mathrm{~cm}^{-1}$ (苯环上 $-\mathrm{C}-\mathrm{H}$ 面外弯曲振动吸收峰)(占新华等, 2007; 肖 彦春和窦森, 2008)。Y0马尾松林芒其覆盖地土壤 DOM红外光谱图在 3 250-3 $750 \mathrm{~cm}^{-1}$ 和 1 $000-$ $1250 \mathrm{~cm}^{-1}$ 均存在一个强而宽的吸收峰(图6), 前者表 明与氢键键合的一 $\mathrm{COOH}$ 、醇、苯酚中的一 $\mathrm{OH}$ 的存 在, 后者表明多糖类、醇类、羧酸类及脂类- $\mathrm{C}-\mathrm{O}-$ 的存在, 这类羟基和一 $\mathrm{C}-\mathrm{O}$ 主要来源于 $\mathrm{DOM}$ 中纤 维素、糖类及淀粉等碳水化合物, 另外还有 $1625-$ $1690 \mathrm{~cm}^{-1}$ 和 $460-680 \mathrm{~cm}^{-1}$ 两个弱而窄的吸收峰以及 1600-1 $425 \mathrm{~cm}^{-1}$ 的弱吸收峰证实了芳香类物质的存 在, 其中特征峰 $3546 \mathrm{~cm}^{-1}$ 和 $3409 \mathrm{~cm}^{-1}$ 的 $\mathrm{N}-\mathrm{H}$ 伸 缩振动以及 $1687 \mathrm{~cm}^{-1}$ 的 $\mathrm{N}-\mathrm{H}$ 的弯曲振动, 证明 酰胺类物质的存在, 然而林下裸地土壤 DOM的特 征峰明显没有芒萁覆盖地的丰富, 它仅有3 250$3750 \mathrm{~cm}^{-1}$ 和 $1000-1250 \mathrm{~cm}^{-1}$ 以及 $460-680 \mathrm{~cm}^{-1}$ 这 几个弱而窄的吸收峰, 并且吸收比例也明显弱于芒 其覆盖地, 去除芒其一年后, 土壤DOM的红外光谱 特征峰与芒其覆盖地差异不明显, 但有接近 $\mathrm{CK}$ 的 趋势; Y31马尾松林土壤DOM红外光谱特征与 $\mathrm{Y} 0$ 年 相同, 林下裸地红光光谱特征峰明显没有芒萁覆盖 地土壤DOM的丰富, 并且吸收比例也要明显低于 芒萁覆盖地, 去除芒其一年后, 土壤DOM红外光谱
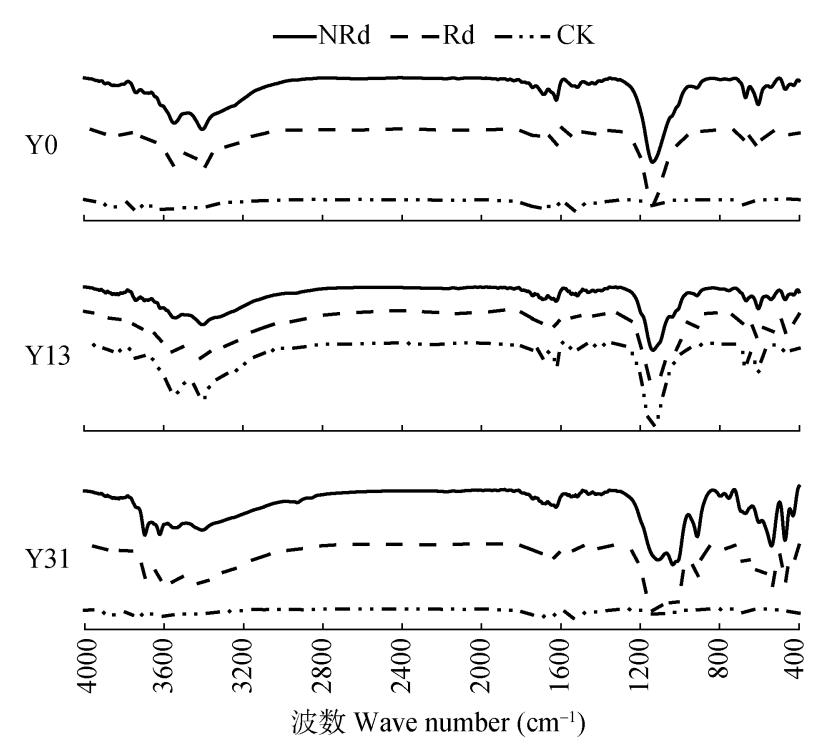

图6 土壤可溶性有机质(DOM)的红外光谱特征。CK, 林下 裸地; NRd, 芒萁覆盖地; Rd, 去除芒萁覆盖地。Y0, 未治理 地; Y13, 恢复13年马尾松林地; Y31, 恢复31年马尾松林地。

Fig. 6 Characteristics of dissolved organic matter (DOM) fourier infrared transmission spectrum. CK, control; NRd, not removed Dicranopteris dichotoma; $\mathrm{Rd}$, removed $D$. dichotoma. Y0, without ecological restoration; Y13, ecological restoration for 13 years; Y31, ecological restoration for 31 years.
特征峰与芒萁覆盖地差异不明显, 但有接近林下裸 地的趋势; 特别的是, Y13马尾松林处理之间土壤 DOM红外光谱的变化趋势并不明显, 芒萁覆盖地、 去除芒萁地和林下裸地土壤DOM光谱上的特征峰 型基本相似, 吸收比例也基本相同, 这可能与Y13 马尾松林处于壮年期, 林分密度大, 林下即使无芒 其覆盖也可获得相当数量和质量的DOM有关。由此 可见, 林下裸地土壤DOM较芒其覆盖地含有更多 着基、羧酸类以及碳水化合物等结构简单且易迁移 的物质, 去除芒萁一年后, 与芒萁覆盖地之间差异 不明显, 这也意味着芒萁可以保持DOM分子的复 杂程度, 维持其稳定性, 但这种影响作用可能是一 个长期缓慢的过程。

\section{3 讨论}

近期的研究发现, 新近调落物和土壤腐殖质分 解以及土壤根系周转及其分泌物和微生物代谢产物 是土壤DOM的主要来源(Chang et al., 2007; Wu et $a l ., 2010$; 刘者等, 2015), 而本研究中马尾松林下裸 地立地条件极差, 其调落物数量、根系周转速度和 分泌物数量以及土壤微生物代谢产物远没有林下芒 萁覆盖地丰富(Xie et al., 2013; 张浩等, 2016), 造成 不同恢复年限马尾松林下芒萁覆盖地土壤 $\mathrm{DOM}$ 数 量显著高于林下裸地 $(p<0.05)$, 去除芒萁覆盖一年 后, 土壤DOM数量降低明显, 说明了林下芒萁是影 响土壤DOM数量的主要因素。与林下裸地相比, 芒 其覆盖下的土壤, 调落物输入的数量和种类更为丰 富, 并且土壤中遍布芒萁的根系, 能够产生大量的 根系分泌物和根系脱落物, 此类活性有机碳恰好是 微生物生命活动的主要能量源(Zhao et al., 2012; 张 浩等, 2016), 而微生物正常生命活动会产生大量微 生物代谢产物。林下芒萁覆盖地土壤DOM在SOC中 的分配比例明显高于林下裸地，且芒其去除一年后， 该比例明显下降, 而DOM作为土壤微生物可直接 分解利用的主要碳源, 其在土壤有机碳中所占的比 例大小表征了土壤可供给微生物能量大小的潜力 (Zhao et al., 2012; 张浩等, 2016), 因此, 芒萁覆盖 地土壤可提供足够的碳源给微生物, 维持其正常的 代谢活动, 当微生物自身代谢所需碳源得到满足后, 土壤有机碳水平便处于动态平衡当中, 由此可见, 芒萁覆盖地土壤有积累土壤有机碳的潜力(Hagedorn, et al., 2000), 即林下芒萁对土壤有机碳积累具有积 
极的作用。

植被类型不同, 土壤DOM结构差异明显(李海 涛等, 2007), 显然, 林下芒萁覆盖地土壤DOM的光 谱学特征与林下裸地不同。本研究的紫外光谱显示, 林下芒萁覆盖地土壤DOM的 $A I$ 显著高于林下裸地, $\mathrm{E}_{2}: \mathrm{E}_{3}$ 值却与之相反, 意味着芒萁覆盖地土壤 $\mathrm{DOM}$ 芳香化程度更高, 且分子量更大, 结构更为复杂 (Chang et al., 2007), 而去除芒萁一年后, 土壤DOM 的 $A I$ 值显著降低, $\mathrm{E}_{2}: \mathrm{E}_{3}$ 值却未见明显变化, 表明芒 萁去除后, 土壤DOM的芳香化程度在降低, 但分子 量变化却不明显。研究发现因芒萁调落物养分含量 低、质量差(Zhao et al., 2012), 所以芒萁群落下常常 覆盖着一层厚厚的芒其枯死物, 其分解后会产生大 量的芳香类物质, 这是导致芒萁覆盖地土壤DOM 芳香化程度较高的主要原因。

如前所述，林下芒萁覆盖地土壤苂光发射光谱 也表明芒萁覆盖地土壤DOM结构中含有更多的苂 光基团, 即腐殖化物质, 并且 Y 31 马尾松土壤DOM 荧光发射光谱特征峰位置发生明显位移, 从裸地到 芒其覆盖地特征峰位置明显向长波方向移动, 说明 芒萁覆盖地土壤DOM的共轭体系增加, 分子结构 更为复杂, 而Y0和Y13马尾松林的这种波峰位置移 动并不明显, 这可能与不同恢复程度的森林生态系 统对于土壤DOM结构变化快慢程度的控制机制不 同有关。芳香环是常见的共轭体系, 它是形成土壤 腐殖质的基础, 腐殖质分子的中心常常是一个稠环 或者易生稠环的芳香核(朱鹤健, 1992), 因而和 $A I$ 的 结果一致。苂光同步光谱结果类似, 芒萁覆盖地土 壤DOM结构中含有更多的蛋白质基团、芳香性脂肪 酸基团和木质素基团, 且去除芒萁一年后, 土壤 DOM各基团特征峰均有不同程度的降低, 证实了 芒萁是土壤DOM结构复杂程度的主要影响因素。许 多研究证实, 林下裸地因其立地条件差, 且缺少林 下植被芒萁覆盖, 所以DOM的输入源(调落物、根系 周转和分泌物、微生物代谢产物)远不如芒萁覆盖地 丰富(Hagedorn et al., 2000; Zhao et al., 2012), 而且 源于芒萁枯落物的DOM中含有许多聚合程度高的 难分解组分, 其分解产物中含有许多结构复杂的大 分子化合物(周国模和姜培坤, 2004), 这不仅有利于 土壤DOM腐殖化程度的提高, 而且有利于其腐殖 质的形成，导致其易被土壤胶体吸附(Kaiser，1997), 增强其化学稳定性, 有利于土壤有机碳的积累。红
外光谱显示, 林下裸地土壤DOM比芒萁覆盖地含 有更多的羟基、羧酸类以及碳水化合物中的烷氧基 等结构简单易迁移的物质, 这与 $A I$ 和 $H I X$ 的规律吻 合, 但芒萁去除一年后, 红外光谱谱图上的特征峰 减少不明显，也说明芒萁对土壤DOM的结构特征 的影响是一个持久缓慢的过程。

\section{4 结论}

不同恢复年限的马尾松林芒其覆盖地, 因其调 落物数量、根系周转和分泌物以及微生物代谢产物 明显多于林下裸地, 故而芒其覆盖地土壤DOM数 量更多, 可供给微生物足够的碳源, 维持土壤有机 碳的动态平衡, 利于土壤有机碳积累。从DOM光谱 学特征判断, 林下芒其覆盖地土壤DOM比林下裸 地的芳香化程度和腐殖化程度更高, DOM的分子量 更大，结构更为复杂，易于被土壤胶粒吸附，维持 其化学稳定, 利于土壤有机碳积累, 即芒萁在土壤 有机碳积累过程中具有明显的积极作用。

基金项目 国家自然科学基金(31370465)和国家重 点基础研究发展计划 (973 计划) 前期专项课题 (2012CB722203)。

\section{参考文献}

Akagi J, Ádám Zsolnay, Bastida F (2007). Quantity and spectroscopic properties of soil dissolved organic matter (DOM) as a function of soil sample treatments: Air-drying and pre-incubation. Chemosphere, 69, 1040-1046.

Andreasson F, Bo B, Bååth E (2009). Bioavailability of DOC in leachates, soil matrix solutions and soil water extracts from beech forest floors. Soil Biology \& Biochemistry, 41, $1652-1658$.

Carter HT, Tipping E, Koprivnjak JF, Millerc PM, Cooksond B, Hamilton-Taylord J (2012). Freshwater DOM quantity and quality from a two-component model of UV absorbance. Water Research, 46, 4532-4542.

Chang SC, Wang CP, Feng CM, Rees R, Hell U, Matzner E (2007). Soil fluxes of mineral elements and dissolved organic matter following manipulation of leaf litter input in a Taiwan Chamaecyparis forest. Forest Ecology \& Management, 242, 133-141.

Fujii K, Uemura M, Hayakawa C, Funakawa S (2009). Fluxes of dissolved organic carbon in two tropical forest ecosystems of East Kalimantan, Indonesia. Geoderma, 152, 127136.

Gielen B, Neirynck J, Luyssaert S, Janssens IA (2011). The importance of dissolved organic carbon fluxes for the carbon balance of a temperate Scots pine forest. Agricultural 
and Forest Meteorology, 151, 270-278.

Hagedorn F, Kaiser K, Feyen H, Schleppi P (2000). Effects of redox conditions and flow processes on the mobility of dissolved organic carbon and nitrogen in a forest soil. Journal of Environmental Quality, 29, 288-297.

Jiao K, Li ZP (2005). Dynamics and biodegradation of dissolved organic carbon in paddy soils derived from red clay. Soils, 37, 272-276. (in Chinese with English abstract) [焦坤, 李忠佩 (2005). 红壤稻田土壤溶解有机 碳含量动态及其生物降解特征. 土壤, 37, 272-276.]

Kaiser K (1997). Dissolved organic matter sorption on subsoil and minerals studied by ${ }^{13} \mathrm{C}-\mathrm{NMR}$ and DRIFT spectroscopy. European Journal of Soil Science, 48, 301-310.

Kalbitz K, Geyer W, Geyer S (1999). Spectroscopic properties of dissolved humic substances-A reflection of land use history in a fen area. Biogeochemistry, 47, 219-238.

Kalbitz K, Kaiser K (2008). Contribution of dissolved organic matter to carbon storage in forest mineral soils. Journal of Plant Nutrition \& Soil Science, 171, 52-60.

Kalbitz K, Meyer A, Yang R, Gerstberger P (2007). Response of dissolved organic matter in the forest floor to long-term manipulation of litter and throughfall inputs. Biogeochemistry, 86, 301-318.

Li HT, Yu GR, Li JY, Chen YR, Liang T (2007). Decomposition dynamics and nutrient release of litters for four artificial forests in the red soil and hilly region of subtropical China. Acta Ecologica Sinica, 27, 898-908. (in Chinese with English abstract) [李海涛, 于贵瑞, 李家永, 陈永 瑞, 梁涛 (2007). 亚热带红壤丘陵区四种人工林调落物 分解动态及养分释放. 生态学报, 27, 898-908.]

Liu Z, Yang YS, Zhu JM, Xie JS, Si YT (2015). Effects of forest conversion on quantities and spectroscopic characteristics of soil dissolved organic matter in subtropical China. Acta Ecologica Sinica, 35, 6288-6297. (in Chinese with English abstract) [刘翥, 杨玉盛, 朱锦禁, 谢锦升, 司友涛 (2015). 中亚热带森林转换对土壤可溶性有机 质数量与光谱学特征的影响. 生态学报, 35, 62886297.]

Mccarthy JF (2005). Carbon fluxes in soil. Journal of Geographical Sciences, 15, 149-154.

Michalzik B, Matzner E (1999). Dynamics of dissolved organic nitrogen and carbon in a Central European Norway spruce ecosystem. European Journal of Soil Science, 50, 579590.

Peuravuori J, Pihlaja K (1997). Molecular size distribution and spectroscopic properties of aquatic humic substances. Analytica Chimica Acta, 337, 133-149.

Schwendenmann L, Veldkamp E (2005). The role of dissolved organic carbon, dissolved organic nitrogen, and dissolved inorganic nitrogen in a tropical wet forest ecosystems. Ecosystems, 8, 339-351.

Wu JS, Jiang PK, Chang SX, Xu QF, Lin Y (2010). Dissolved soil organic carbon and nitrogen were affected by conver- sion of native forests to plantations in subtropical China. Canadian Journal of Soil Science, 90, 27-36.

Xiao YC, Dou S (2008). Study on infrared spectra of soil humus fractions. Chinese Journal of Analytical Chemistry, 35, 1596-1600. (in Chinese with English abstract) [肖彦 春, 窦森 (2008). 土壤腐殖质各组分红外光谱研究. 分 析化学, 35, 1596-1600.]

Xie JS, Guo JF, Yang ZJ, Huang ZQ, Chen GS, Yang YS (2013). Rapid accumulation of carbon on severely eroded red soils through afforestation in subtropical China. Forest Ecology \& Management, 300, 53-59.

Yang YS, Guo JF, Chen GS, Chen YX, Yu ZY, Liu DX (2003). Origin, property and flux of dissolved organic matter in forest ecosystems. Acta Ecologica Sinica, 23, 547-558. (in Chinese with English abstract) [杨玉盛, 郭剑芬, 陈光水, 陈银秀, 于占源, 董涁, 刘东霞 (2003). 森林生态系统 DOM的来源、特性及流动. 生态学报, 23, 547-558.]

Zhan XH, Zhou LX, Yang H, Jiang TH (2007). Infrared spectroscopy of DOM-PAHs complexes. Acta Pedologica Sinic, 44, 47-53. (in Chinese with English abstract) [占新 华, 周立祥, 杨红, 蒋廷惠 (2007). 水溶性有机物与多 环芳烃结合特征的红外光谱学研究. 土壤学报, 44, 47-53.]

Zhang H, Lyu MK, Jiang J, Pu XT, Wang EX, Qiu X, Xie JS (2016). Effect of vegetation restoration on topsoil and subsoil organic carbon mineralization in red soil erosion area, Journal of Soil and Water Conservation, 30, 244-249. (in Chinese with English abstract) [张浩, 吕茂 奎, 江军, 蒲晓婷, 王恩熙, 邱曦, 谢锦升 (2016). 侵蚀 红壤区植被恢复对表层与深层土壤有机碳矿化的影响. 水土保持学报, 30, 244-249.]

Zhao J, Wan S, Li Z, Shao Y, Xu G, Liu Z, Zhou L, Fu S (2012). Dicranopteris-dominated understory as major driver of intensive forest ecosystem in humid subtropical and tropical region. Soil Biology \& Biochemistry, 49, 78-87.

Zhou GM, Jiang PK (2004). Changes in active organic carbon of erosion red soil by vegetation recovery. Journal of Soil and Water Conservation, 18, 68-70. (in Chinese with English abstract) [周国模，姜培坤 (2004). 不同植被恢 复对侵蚀型红壤活性碳库的影响. 水土保持学报, 18 , 68-70.]

Zhou JM, Dai JY, Pan GX (2004). Fractionation and spectroscopic property of dissolved organic matters in soils. Spectroscopy and Spectral Analysis, 24, 1060-1065. (in Chinese with English abstract) [周江敏, 代静玉, 潘根兴 (2004). 应用光谱分析技术研究土壤水溶性有机质的分 组及其结构特征. 光谱学与光谱分析, 24, 1060-1065.]

Zhu HJ (1992). Soil Geography. Higher Education Press, Beijing. (in Chinese) [ 朱鹤健 (1992). 土壤地理学. 高等教 育出版社, 北京.]

责任编委: 程晓莉 责任编辑: 王 藏 


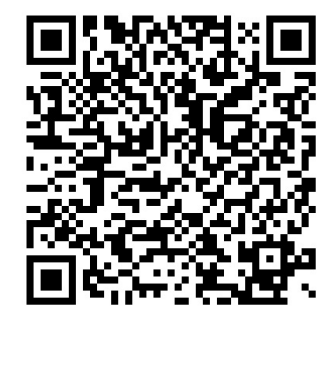

